\title{
Comparative Study between Various PAPR Reduction Technique
}

\author{
Sahil Malik ${ }^{1}$, Ruchi Sharma \\ Department of Electronics and Communication Engineering Vivekananda Global University, Jaipur, Rajasthan, \\ India.
}

\begin{abstract}
This paper presents the comparative study between various PAPR reduction technique such as partial transmit sequence, DFT spreading, Clipping and filtering. The peak to average power ratio of the time domain envelope is an important parameter at the physical layer of the communication system using OFDM signaling. The signals must maintain a specified average energy level in the channel to obtain the desired Bit-error- rate. The peak signal level relative to that average defines the maximum dynamic range that must be accommodated by the components in the signal flow path to support the desired average. High Peak to Average Power Ratio (PAPR) for OFDM system is still a demanding area and difficult issue. The radio transmitter stations for covering and getting enough transmitted power in their desired area has to use High Power Amplifier (HPA). If the high power amplifier doesn't work in linear region, it can cause the out-of-band power to be kept under the specified limits. This condition can cause inefficient amplification and expensive transmitters, thus it is necessary to investigate PAPR reduction techniques for OFDM system. Some of the performance measures like Complementary Commutative Distribution Function of PAPR and BER are analyzed through simulation to demonstrate the efficacy of the various scheme.
\end{abstract}

\section{INTRODUCTION}

Mobile radio communication systems are progressively required to give a variety of brilliant multi-media services to mobile users. To meet these demands in remote broadband communication frameworks must have the capacity to bolster high limit, variable bit rate data transmission and high data transfer capacity effectiveness. In the mobile radio condition, signals are normally disabled by fading and what's more, multi-path delay phenomenon. In such channels, extreme fading of the signal amplitude and inter symbol interference (ISI) because of the frequency selectivity of the channel cause an unsuitable degradation of the error performance. Orthogonal frequency division multiplexing (OFDM) is a multi-carrier modulation scheme for high data rate mobile communication system because of its vigor to frequency selective fading, high spectral efficiency and low computational complexity. Recently OFDM system has been getting across the board consideration. Orthogonal frequency division multiplexing can be used in conjunction with multiple-input multiple-output (MIMO) method to enhance diversity gain and/or the system capacity by exploiting spatial domain. MIMO-OFDM is viewed as a key innovation in rising high data-rate communication system, including digital subscriber lines (DSL), IEEE 802. 11, IEEE 802.16 and IEEE 802.15.3a, give a course to $4 \mathrm{G}$ technology that support high mobile data rates. The primary advantages of utilizing MIMO-OFDM technique, which can diminish the recipient unpredictability and manage the multipath fading effectively, incorporate high spectral efficiency and robustness against narrowband. The fundamental points of interest of utilizing MIMO-OFDM procedures, which can diminish the recipient intricacy and manage the multipath fading effectively, incorporate high spectral efficiency and strength against narrowband interference, uniform average spectral density, capacity of dealing with exceptionally solid echoes, less non-linear distortion and efficient implementation. Notwithstanding, the principle limitation of utilizing MIMO-OFDM endures with the problem of high PAPR and carrier frequency offset sensitivity [6]. Subsequently, it is imperative to decrease the PAPR; otherwise, high power amplifier (HP A) in the transmitter need to have a linear region that is substantially bigger than the average power, which makes them costly and wasteful. This on the because if HPA with a linear region somewhat more noteworthy than the average power is utilized, the saturation brought about by the vast peak will result in intermodulation distortion, which expands the bit error rate (BER) and causes spectral widening, bringing about adjacent channel interference. A number of PAPR reduction techniques have been proposed to handle this problem. One of the most widely used strategies is Clipping and Filtering, DFT Spreading and Partial Transmit sequence (PTS), utilizing probabilistic techniques [3]. The guideline of probabilistic strategy relies on upon decreasing the likelihood of high PAPR by creating 
several OFDM symbols (multiple candidates) conveying a similar information and choosing the one having the most minimal PAPR [2]. The probabilistic technique can likewise be classified into two systems: sub block partitioning methodology and entire block procedure. The sub block partitioning procedure, for example, partial transmit sequence (PTS) [8-9], separates frequency domain signals into several sub blocks. on the other hand, the entire block Strategy, for example, Clipping and Filtering, DFT Spreading, [5-8] consider the whole block for creating numerous candidates. To begin with, the entire block procedure of the probabilistic strategies to create numerous candidates is viewed as, and then the probability Distribution Function (PDF) for the multiple candidate system is investigated. When the candidate having the reduced APR is chosen, the PDF of the amplitude of a selected OFDM symbol becomes a function of the number of candidates [1-4]. All through this paper we portray the fundamental rule of every one of these procedures. The determination of any of the PAPR reduction technique may be at the cost of PAPR reduction ability, synchronization between the transmitter and the receiver. The absence of the PAPR reduction techniques will cause the increase in the transmit power, bit error rate at the receiver, the data rate loss, and the computational complexity. Here we have studied through simulation result the performance of PTS and Clipping and Filtering, DFT Spreading based PAPR reduction techniques for these techniques in view of different parameters.

\section{PRINCIPLE ALGORITHM FOR REDUCING PAPR:}

An OFDM signal includes various independently modulated sub-carrier, which can give a considerable PAPR when added up coherently. At the point when $\mathrm{N}$ signals are added with the similar phase, they generate a peak power that is $\mathrm{N}$ times the average power of the signal. So OFDM signal has a substantial PAPR, which is sensitive to non-linearity of the high power amplifier. PAPR is a historic issue in the advancement of the Wireless communication, the more PAPR of OFDM the more prerequisites and difficulties for executing the HPA. However the PAPR is computed from the peak amplitude of the waveform divided by the average value of waveform as follows:

$$
\mathrm{PAPR}=\frac{\operatorname{maX}_{0} \leq t \leq n T\left|X_{n}\right|^{2}}{E\left\{\left|X_{n}\right|^{2}\right\}}
$$

Where $E\left\{|x(t)|^{2}\right\}$ is average power of the waveform. The amplitude of the waveform has Rayleigh distribution. The distribution of PAPR states in term complementary cumulative distribution function (CCDF).

Assume $\mathrm{PAPR}_{0}=\mathrm{A}$

$$
\mathrm{CCDF}=\operatorname{Pr}\left(\mathrm{PAPR}<\mathrm{PAPR}_{0}=\mathrm{A}\right)
$$

$\mathrm{F}_{\mathrm{X}}(\mathrm{PAPR})=\mathrm{Pr}$

$\left[\frac{\operatorname{maX}_{0} \leq t \leq n T\left|X_{n}\right|^{2}}{E\left\{\left|X_{n}\right|^{2}\right\}} \leq P^{2} A P R_{0}\right]$

$=1-\exp \left(-\mathrm{PAPR}_{0}\right)$

$\mathrm{CCDF}=\operatorname{Pr}\left(\mathrm{PAPR}>\mathrm{PAPR}_{0}\right)=1 \mathrm{~F}_{\mathrm{X}}\left(\mathrm{PAPR}_{0}\right)^{\mathrm{N}}$

$=1-\left(1-\exp \left(-\mathrm{PAPR}_{0}\right)\right)^{\mathrm{N}}$

The decibel form of equation (4) is as following:

$\mathrm{PAPR}_{\mathrm{dB}}=10 \log _{10}(\mathrm{PAPR})$

Despite the fact that the probability of the largest PAPR to happen is not high, but rather to send the high PAPR of OFDM signal with no distortion, the probability increases. All the linearity in High Power Amplifier (HPA) and A/D converter ought to meet the necessities said above [1-3]. Since the hardware that meets these necessities is exceptionally costly, therefore, it is extremely requesting and essential to reduce PAPR in OFDM system [9-10].

\subsection{PAPR and its Reduction in OFDM System}

A block diagram of OFDM system is delineated in Fig. 1. The OFDM flag comprises of an sum of subcarriers that are modulated by phase shift keying (PSK) or Quadrature Amplitude modulation (QAM) . To start with, the serial input data stream is arranged into $\mathrm{N}$ group of bits, where $\mathrm{N}_{\mathrm{c}}$ is the number of subcarriers. The number of bits in each of the $\mathrm{N}_{\mathrm{c}}$ group decides the constellation size mea for that specific subcarrier. The mapped complex symbol are then serial-to-parallel (S/P) converted and oversampled by a factor $\mathrm{L}$ resulting in a block $\mathrm{N}_{\mathrm{c}} \mathrm{L}$ complex symbols [11]. 


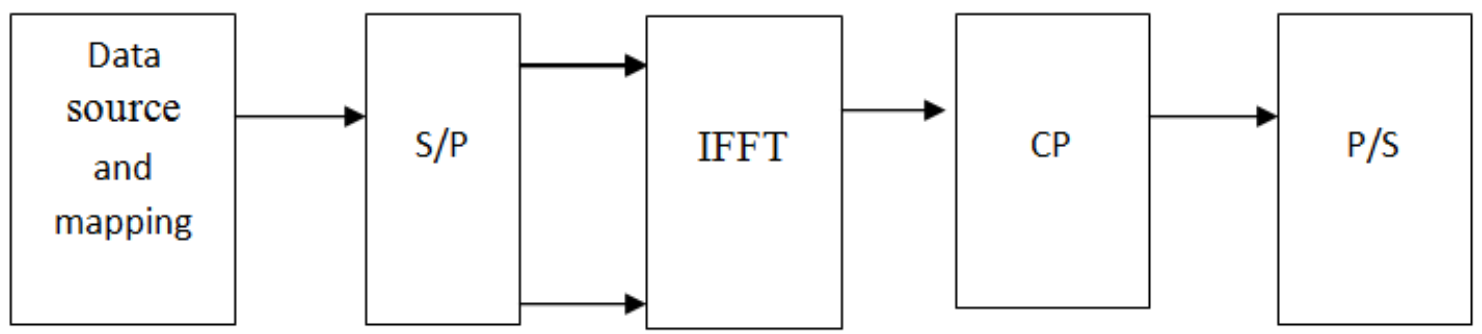

Figure1. Block diagram of OFDM system.

The complex discrete time base band OFDM signal can be expressed as:

$$
X_{N_{T}}(t)=\frac{1}{\sqrt{N}} \sum_{n=0}^{N-1} X_{n} e^{j 2 \pi F_{n} t}, 0 \leq t \leq n T
$$

Where $\mathrm{N}_{\mathrm{T}}$ transmit an antenna that make use of $\mathrm{N}$ subcarrier. By using OFDM modulation technique, a block of $\mathrm{n}$ data symbols (one OFDM symbol) $\left\{X_{n}, n=0,1 \ldots n-1\right\}$ will be transmitted in parallel such that each symbol modulate a different carrier such that each modulates a different subcarrier from a set $\left\{\mathrm{f}_{\mathrm{N}}, \mathrm{N}=0,1 \ldots \mathrm{N}-1\right\}$. The $\mathrm{N}$ subcarriers are orthogonal, that is $\mathrm{f}_{\mathrm{N}}=\mathrm{N} \Delta \mathrm{f}$ where $\Delta \mathrm{f}=1 / \mathrm{nT}$ and $\mathrm{T}$ is the symbol time period. The above power attributes can likewise be portrayed regarding their magnitude (not power) by defining the crest factor $(\mathrm{CF})$ as:

Pass Band and Condition: $\mathrm{CF}=\sqrt{P A P R}$

In the PSK/OFDM system with $\mathrm{N}$ subcarriers, the most extreme power happens when the greater part of the $\mathrm{N}$ subcarrier components happen to be added with same phase. Assuming that $\mathrm{E}\left\{\left|\mathrm{X}_{\mathrm{n}}\right|^{2}\right\}=1$. It result in $\mathrm{PAPR}=\mathrm{N}$, that is the maximum power equivalent to $\mathrm{N}$ times the average power. We have noticed that when M-QAM is greater than 4 times M-PSK, the PAPR will be large in this case. The Probability distribution of $X_{n}$ will follow after the Gaussian distribution. The amplitude of OFDM signal has a Rayleigh distribution with zero mean and a variance of $\mathrm{N}$ times the variance of one complex sinusoid. let $\{\mathrm{Zn}\}$ be chance to be the extents of complex samples. Accepting that the average power of complex pass band OFDM signal $X_{n}$ is equivalent to one, the $\{\mathrm{Zn}\}$ are the standardized Rayleigh arbitrary variable with its own average power, which has the probability density function [4-9] as shown below:

$$
F_{z_{n}}(Z)=\frac{Z}{\sigma^{2}} e^{-Z^{2}} / 2 \sigma^{2}=2 Z e^{-Z^{2}}
$$

Where $\mathrm{E}\left\{\left|\mathrm{Z}_{\mathrm{n}}\right|^{2}\right\}=2 \sigma$.

\subsection{PAPR Reduction Techniques:}

\section{A. Partial transmit sequence}

The Partial Transmit Sequence has been presented by Muller and Hubber in 1997 [14-15]. The PTS technique is based on phase shifting of sub-block of data and then multiplication of data structure by random vectors. This technique is flexible and efficient for OFDM framework. A block diagram of PTS technique is shown in figure 2. In partial transmit sequence [12-13] the input data block of $\mathrm{N}$ symbols is further divided into $\mathrm{V}$ disjoint sub-blocks. The input data block can be written in form of $\mathrm{X}_{\mathrm{V}}$ as:

$$
\mathrm{X}=\sum_{V=0}^{V-1} X_{V}
$$

Each partitioned sub-block is multiplied by a corresponding complex phase factor $\mathrm{b}^{v}=\mathrm{e}^{\mathrm{j} \Phi_{v}}, v=$ $1,2, \ldots . \mathrm{V}$ then taking its IFFT to yield

$$
\begin{gathered}
\mathrm{X}=\operatorname{IFFT}\left\{\sum_{v=1}^{V} b^{v} X^{v}\right\}=\sum_{v=1}^{V} b^{v} \operatorname{IFFT}\left\{X^{v}\right\} \\
=\sum_{v=1}^{V} b^{v} X^{v}
\end{gathered}
$$

Where $X^{v}$ represent the partial transmit sequence. The value of phase vector is selected in such a manner that the value of resulted PAPR is minimized [8]. This is shown as

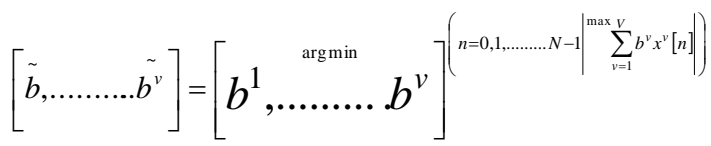

Then the corresponding time domain signal with the lowest PAPR can be expressed as

$$
\mathrm{X}=\{\}
$$

In general the reduction in search complexity largely depends upon the selection of phase factor which is limited to a set of elements. As the set of allowed phase factors is

$$
b=\left\{e^{j 2 \Pi i / w} i=0,1,2, \ldots w-1\right\}, \mathrm{w}^{\mathrm{v}-1}
$$

Therefore search complexity is directly proportional to the number of sub-block. 


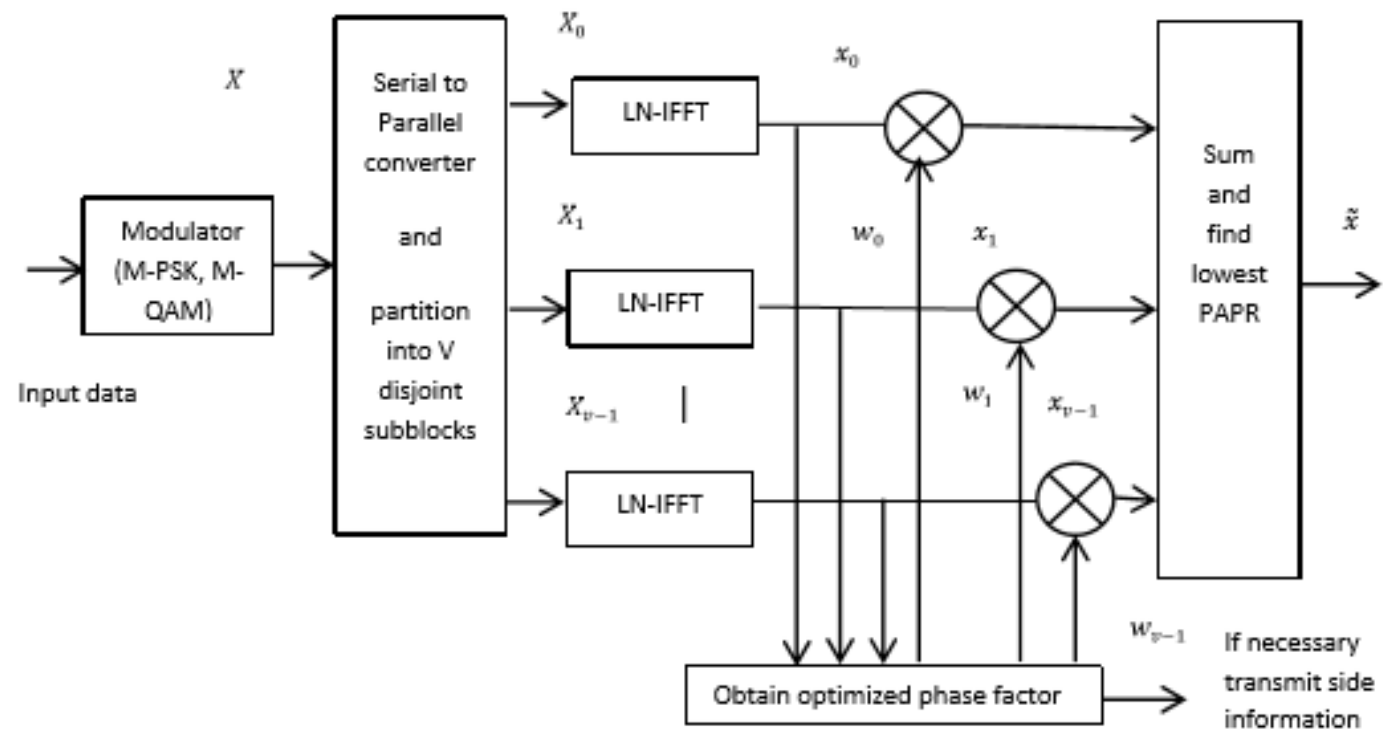

Figure2: A block diagram of PTS technique.

\section{B. Clipping and Filtering}

High PAPR is a standout amongst the most widely recognized issues in OFDM. A high PAPR brings about limitations like increased complexity of the ADC and DAC and furthermore decreased efficiency of radio frequency (RF) power amplifier. One of the straightforward and, viable PAPR reduction strategies is clipping, which cancels out the signal component that exceed some constant amplitude called clip level. In any case, clipping yields distortion power, which is called clipping noise, and expand the transmitted signal spectrum, which causes interference. Clipping is nonlinear process and causes in-band noise distortion, which causes degradation in the performance of bit error rate (BER) and out-of- band noise, which decrease the spectral efficiency [15] . Clipping and filtering technique is powerful in eliminating components of the expanded spectrum. Although filtering can diminish the spectrum development, filtering subsequent to clipping can decrease the out-of-band radiation, yet may likewise bring about some peak re-growth, which the peak signal exceed in the clip level. The strategy of iterative clipping and filtering diminishes the PAPR without spectrum expansion. However, the iterative signal takes long time and it will increase the computational complexity of an OFDM transmitter. A block diagram of PAPR reduction scheme using clipping and filtering is shown in figure 3 [16]. Here $\mathrm{N}$ is the number of subcarrier.

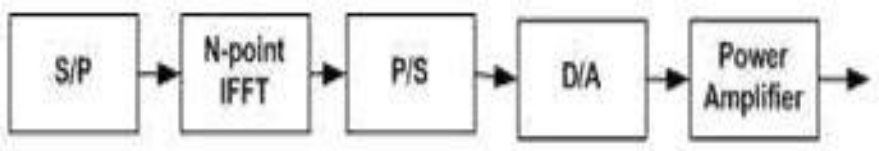

(a)

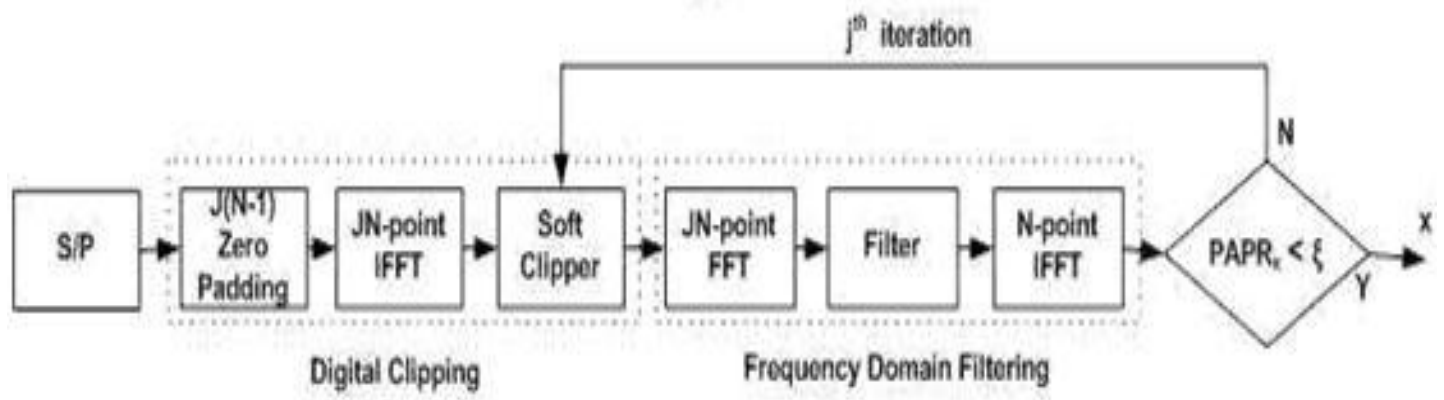

(b)

Figure 3: (a) OFDM system (b) Clipping and Filtering Block diagram. 


\section{DFT Spreading}

DFT -Spreading stands for Discrete

Fourier Transform spreading, it involves the spreading of input signal with the help of DFT (Discrete Fourier Transform) operation which can be taken into IDFT. DFT Spreading results in reduction of PAPR of OFDM signal to the extent of single carrier transmission. Mobile terminal in uplink transmission make use of this technique. It is called as single carrier FDMA. The single carrier FDMA (SC-FDMA) is used for uplink transmission in 3GPP LTE standard [15]. DFT of identical size as IDFT is used as (spreading code). Then OFDMA system becomes equivalent to SCFDMA system. The reasons being DFT and IDFT operations cancel each other. In this case the PAPR of transmitted signal will be same as single carrier system. In OFDMA framework, subcarrier are partitioned and allocated to multiple mobile user. In this case each terminal in uplink uses a subset of subcarrier to transmit its own data whereas it does not happen in case of downlink. The remaining subcarriers which are not used for their own transmission will be filled with zeros. The effect of PAPR reduction depends on the way of assuming the subcarrier to each terminal [6]. Here the input data $x[\mathrm{~m}]$ is DFT spread to produce $X[\mathrm{i}]$ and then allocated as

$$
\tilde{x}[k]=\left\{\begin{array}{ll}
X[k / s] & k=s \cdot m 1, m 1=0,1,2 \ldots \ldots . M-1 \\
0 & \text { otherwise }
\end{array}\right\}
$$

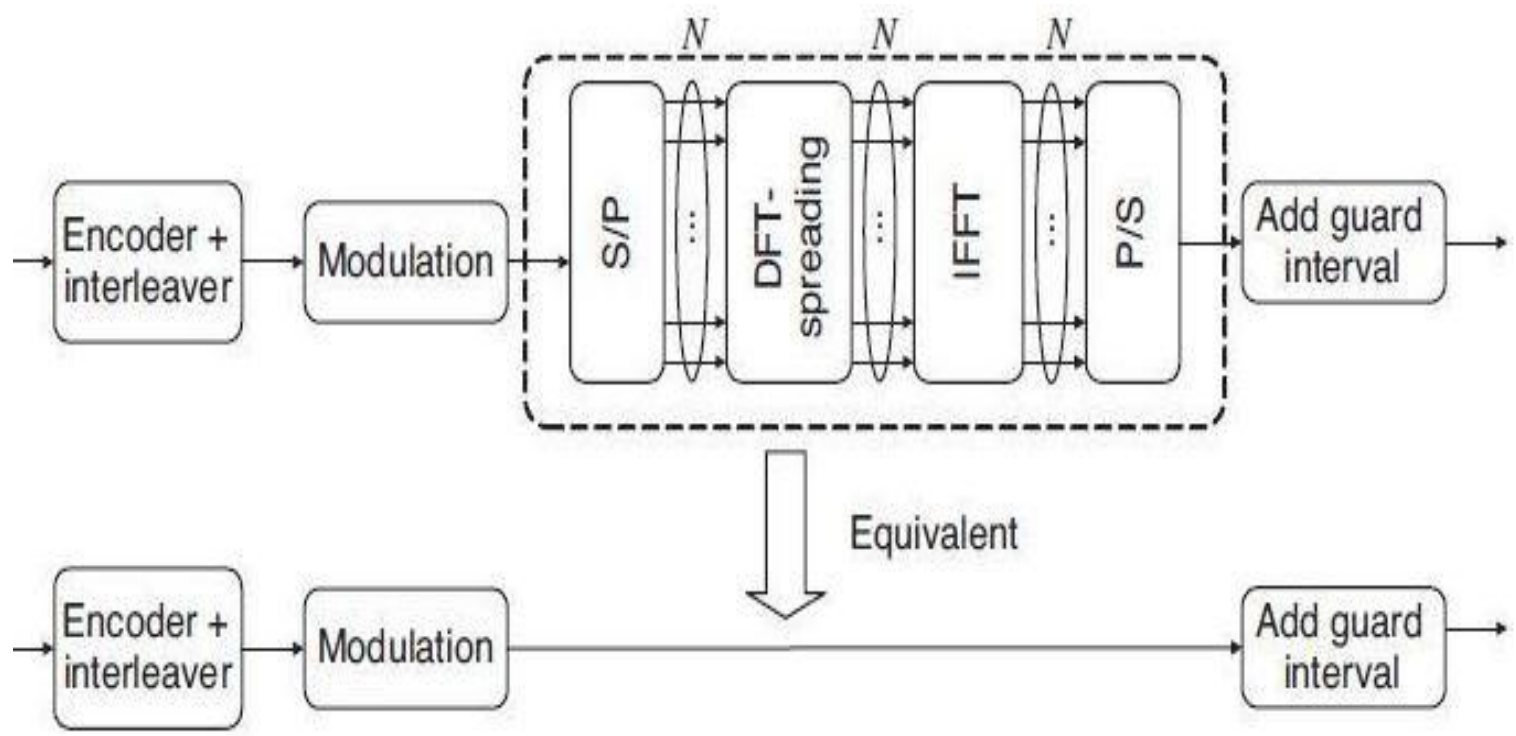

Figure 4: Equivalent of OFDMA system with DFT spreading code to a single-carrier system [15]

\section{SIMULATION RESULT}

\subsection{Partial Transmit Sequence}

We realized from the above discussion that in PTS [13-16\&17-18] approach, there are varying parameters that impact the PAPR reduction performance, these are:

1) The number of sub-blocks $V$, which influences the complexity strongly;

2) The number of possible phase value $W$, which impacts the complexity as well.

In our simulation, two parameters will be considered. They are sub-block sizes $\mathrm{V}$ and different sub-block partition proposals.
The number of computations for Eq. in this suboptimal combination algorithm is $\mathrm{V}$, which is much fewer than that required by the original PTS technique (i.e. $V \square W^{V}$. Figure 5.2 shows the CCDF of PAPR for a 16 QAM/OFDM system using PTS technique as the number of subblock varies. It is seen that the PAPR performance Improves as the number of subblocks increases with $\mathrm{V}=1,2,4,8,16$ and 32 . 


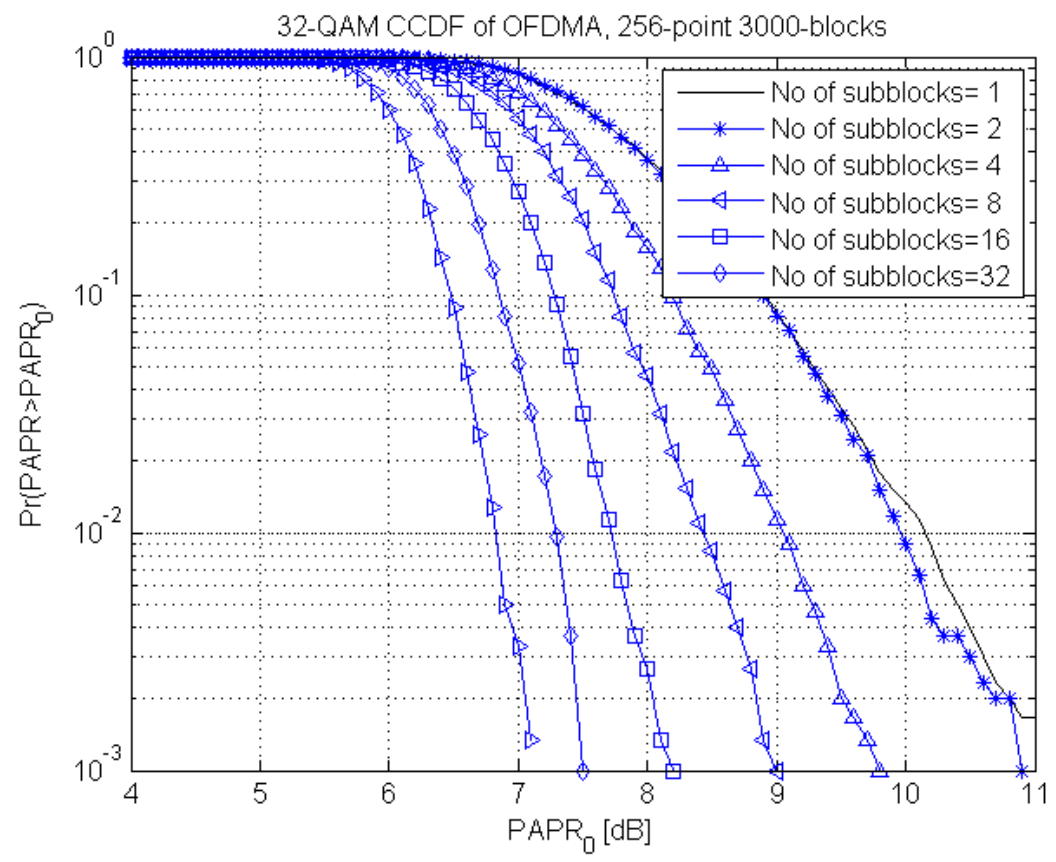

Fig.5: PAPR performance of a 32-QAM/OFDM system with PTS technique when the number of Sub blocks Varies.

\subsection{Clipping and Filtering}

Figure 5.3(a) shows the CCDFs of crest factor $(\mathrm{CF})$ for the clipped and filtered OFDM signals. Recall that the CCDF of $\mathrm{CF}$ can be considered as the distribution of PAPR since CF is the square root of PAPR. It can be seen from this figure that the PAPR of the OFDM signal decreases significantly after clipping and increases a little after filtering. Note that the smaller the clipping ratio (CR) is, the greater the PAPR reduction effect is. Figure 5.3(b) shows the BER performance when clipping and filtering technique is used. Here, "C" and "C\&F" denote the case with clipping only and the case with both clipping and filtering, respectively. It can be seen from this figure that the BER performance becomes worse as the $\mathrm{CR}$ decreases.

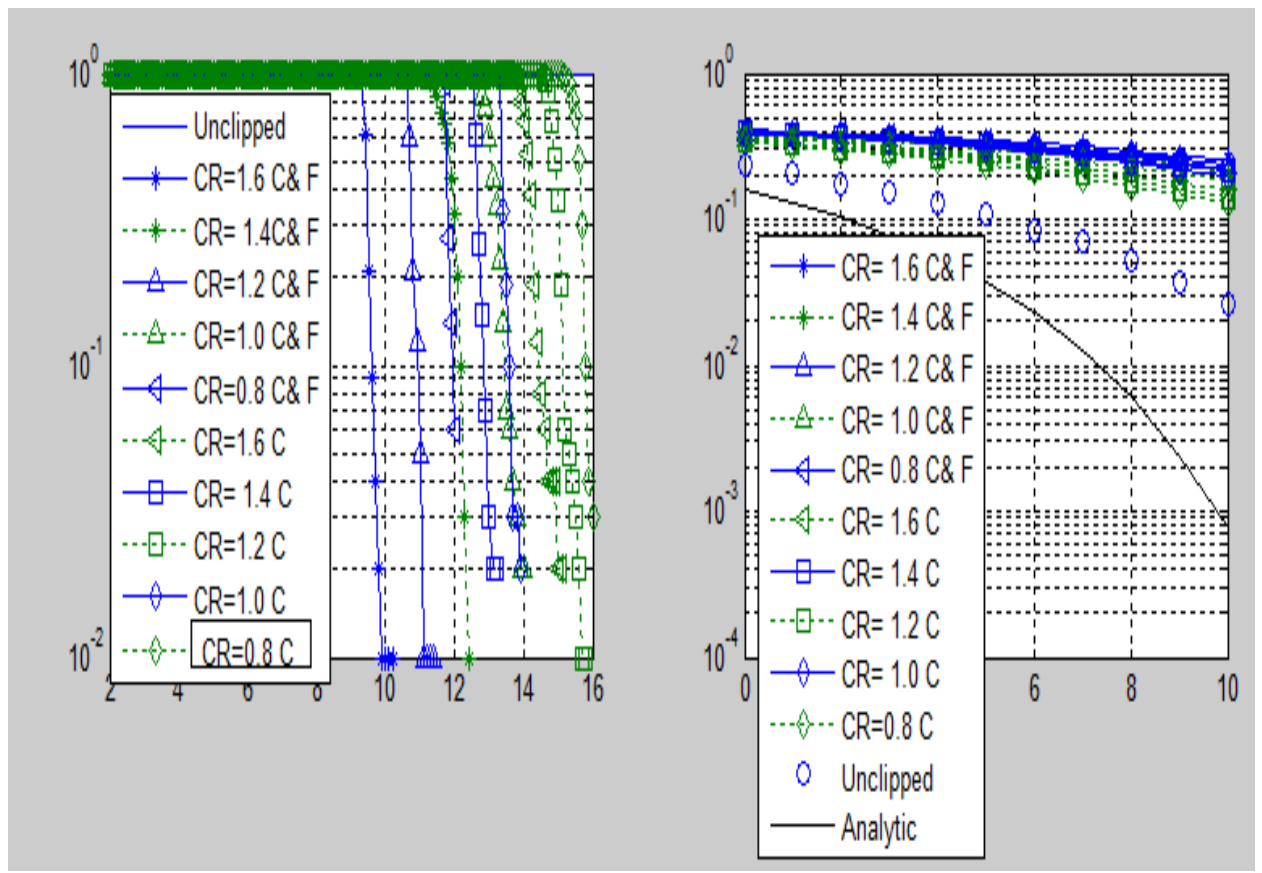

Fig.5.3 PAPR distribution and BER performance with clipping and filtering. 


\subsection{DFT Spreading}

Figure 5.4 shows a comparison of PAPR performances when the DFT-spreading technique is applied to the IFDMA, LFDMA, and OFDMA. Here, QPSK, 16-QAM, and 64-QAM are used for an SC-FDMA system with $\mathrm{N}=256, \mathrm{M}=64$ and $\mathrm{S}=4$. It can be seen from Figure 5.4 that the PAPR performance of the DFT-spreading technique varies
Depending on the subcarrier allocation method. In the case of 16-QAM, the values of PAPRs with IFDMA, LFDMA, and LFDMA for CCDF of $1 \%$ are $3.5 \mathrm{~dB}, 8.3 \mathrm{~dB}$, and $10.8 \mathrm{~dB}$, respectively. It implies that the PAPRs of IFDMA and LFDMA are lower by $7.3 \mathrm{~dB}$ and $3.2 \mathrm{~dB}$, respectively, than that of OFDMA with no DFT spreading.
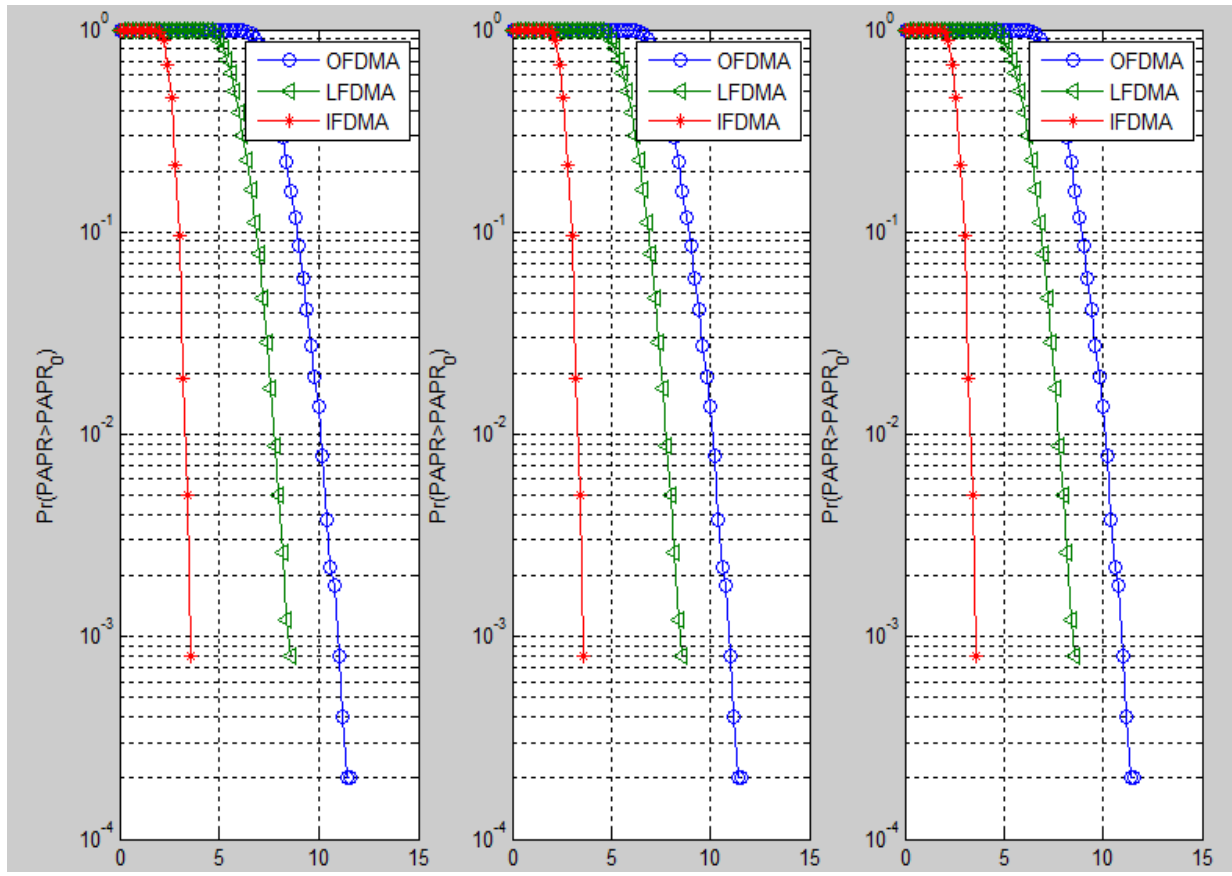

Fig.5.4 PAPR performances of DFT-spreading technique for IFDMA, LFDMA, and OFDMA.

\section{CONCLUSION}

In This paper we analyzed the performance of DFT spread OFDM method for reducing the PAPR in OFDM. The simulation results shows IFDMA method is best among the OFDMA, LFDMA and IFDMA. But the disadvantage of IFDMA scheme is that we are losing user diversity and investigates one of the bottleneck problems that exist in OFDM wireless communication system. High Peak Average Power Ratio (PAPR of OFDM signal), and discusses how to reduce it by different effective system.

\section{REFERENCE}

[1]. van Nee, R.D.J. (Nov. 1996) OFDM codes for peak-toaverage power reduction and error correction. IEEE GTC, London, vol. 2, pp. 740-744.

[2]. Davis, J.A. and Jedwab, J. (1999) Peak-tomean power control in OFDM, Golay complementary sequences, and Reed-Muller codes. IEEE Trans. Info. Theory, 45(7), 2397-2417.

[3]. Davis, J.A. and Jedwab, J. (1997) Peak-tomean power control and error correction for
OFDM transmission using Golay sequences and Reed-Muller codes. Electron. Lett., 33(4), 267-268.

[4]. Urbanke, R. and Krishnakumar, A.S. (Oct. 1996) Compact description of Golay sequences and their extensions. Proc.of the Thirty-Fourth Annual Allerton Conference on Commun., Control and Computing Pagination, Urbana, IL, pp. 693-701.

[5]. Li, X. and Ritcey, J.A. (1997) M-sequences for OFDM peak-to-average power ratio reduction and error correction. Electron. Lett., 33(7), 554-555.

[6]. Tellambura, C. (1997) Use of m-sequences for OFDM peak-to-average power ratio reduction. Electron. Lett., 33(15), 13001301.

[7]. Park, M.H. et al. (2000) PAPR reduction in OFDM transmission using Hadamard transform. IEEE ICC'00, vol. 1, pp. 430433.

[8]. Bauml, R.W., Fischer, R.F.H., and Huber, J.B. (1996) Reducing the peak-to-average power ratio of multicarrier modulation by 
selective mapping. Electron. Lett., 32(22), 2056-2057.

[9]. hkubo, N. and Ohtsuki, T. (Apr. 2003)

Design criteria for phase sequences in selected mapping. IEEE VTC'03, vol. 1, pp. 373-377.

[10]. Muller, S.H. et al. (1997) OFDM with reduced peak-toaverage power ratio by multiple signal representation. In Annals of Telecommun., 52(1-2), 58-67.

[11]. Mahendra Kumar et. Al." Performance Improvement of MIMO-OFDM System PAPR Reduction using pulse shaping", International Journal of Science \& Research (IJSR), Vol. 4, No. 7, July. 2015, pp.23-28. 